\title{
ALGEBRAIC CYCLES ON COMPACT QUATERNIONIC SHIMURA FOURFOLDS AND POLES OF $L$-FUNCTIONS
}

\author{
CRISTIAN VIRDOL \\ Department of Mathematics, Columbia University, Room 509, MC 4406, 2990 Broadway, \\ New York, NY 10027, USA \\ e-mail:virdol@math.columbia.edu
}

(Received 8 June 2010; accepted 25 August 2010; first published online 9 December 2010)

\begin{abstract}
In this article we prove Tate conjecture for a large class of compact quaternionic Shimura fourfolds.

2010 Mathematics Subject Classification. 11F41, 11F80, 11R42, 11R80, 14C25.
\end{abstract}

1. Introduction. Let $X$ be a smooth projective variety of dimension $n$ defined over a number field $F$ and let

$$
\bar{X}=X \times_{F} \overline{\mathbb{Q}} .
$$

For a prime number $l$, let $H_{\mathrm{et}}^{i}\left(X, \overline{\mathbb{Q}}_{l}\right)$ be the étale cohomology of $\bar{X}$. If $K$ is a number field, we denote $\Gamma_{K}:=\operatorname{Gal}(\overline{\mathbb{Q}} / K)$. The Galois group $\Gamma_{F}$ acts on $H_{\mathrm{et}}^{i}\left(X, \overline{\mathbb{Q}}_{l}\right)$ by a representation $\phi_{i, l}$. For any $j \in \mathbb{Z}$, let $H_{\mathrm{et}}^{i}\left(X, \overline{\mathbb{Q}}_{l}\right)(j)$ denote the representation of $\Gamma_{F}$ on $H_{\text {et }}^{i}\left(X, \overline{\mathbb{Q}}_{l}\right)$ defined by $\phi_{i, l} \otimes \xi_{l}^{j}$, where $\xi_{l}$ is the $l$-adic cyclotomic character. For any finite extension $E / F$ the elements of $V^{i}(X, E):=H_{\mathrm{et}}^{2 i}\left(X, \overline{\mathbb{Q}}_{l}\right)(i)^{\Gamma_{E}}$ are called Tate cycles on $X$ defined over $E$. The union

$$
V^{i}(X):=\cup_{E} V^{i}(X, E)
$$

is the space of all Tate cycles on $X$.

To each algebraic subvariety $Y$ of $X$ of codimension $i$, one can associate a cohomology class

$$
[Y] \in H_{2 n-2 i}(X(\mathbb{C}), \mathbb{Q}) \cong H_{B}^{2 i}(X(\mathbb{C}), \mathbb{Q})(i),
$$

where $H_{B}^{2 i}(X(\mathbb{C}), \mathbb{Q})$ is the Betti cohomology. Then using the isomorphism

$$
H_{B}^{2 i}(X(\mathbb{C}), \mathbb{Q})(i) \otimes_{\mathbb{Q}} \mathbb{Q}_{l} \cong H_{\mathrm{et}}^{2 i}\left(X, \mathbb{Q}_{l}\right)(i),
$$

we obtain a class $[Y] \in H_{\mathrm{et}}^{2 i}\left(X, \mathbb{Q}_{l}\right)(i)$. A cohomology class $[Y]$ obtained in this way is called algebraic. If $Y$ is defined over a finite extension $E$ of $F$, then we obtain a class $[Y] \in H_{\text {et }}^{2 i}\left(X, \mathbb{Q}_{l}\right)(i)^{\Gamma_{E}}$. Let $U^{i}(X, E)$ be the space of algebraic cycles defined over $E$. Then $U^{i}(X, E) \subseteq V^{i}(X, E)$ and the first part of the Tate conjecture [16] states that for any finite extension $E / F$ we have

$$
U^{i}(X, E)=V^{i}(X, E),
$$

i.e. every Tate cycle is algebraic. 
The $L$-function $L^{2 i}\left(s, X_{/ F}\right)$ (more exactly the Euler product) attached to the representation $\phi_{2 i, l}$ converges for $\operatorname{Re}(s)>i+1$. The second part of the Tate conjecture [16] states that for any finite extension $E / F$ the $L$-function $L^{2 i}\left(s, X_{/ E}\right)$ has a meromorphic continuation to the entire complex plane and has a pole at $s=i+1$ of order equal to

$$
\operatorname{dim}_{\overline{\mathbb{Q}}_{l}} V^{i}(X, E) .
$$

We consider a quartic totally real number field $F$ containing a quadratic subfield. Let $B$ be a quaternion division algebra over $\mathbb{Q}$ and let $D:=B \otimes_{\mathbb{Q}} F$. We assume that $D$ is a quaternion division algebra over $F$ which splits at the real places. Let $G$ be the algebraic group over $F$ defined by the multiplicative group $D^{\times}$of $D$ and let $\bar{G}=\operatorname{Res}_{F / \mathbb{Q}}(G)$. We denote by $S_{K}:=S_{\bar{G}, K}$ the canonical model of the quaternionic Shimura variety associated with an open compact subgroup $K$ of $\bar{G}\left(\mathbb{A}_{f}\right)$, where $\mathbb{A}_{f}$ is the finite part of the ring of adeles $\mathbb{A}_{\mathbb{Q}}$ of $\mathbb{Q}$. Then, $S_{K}$ is a four-dimensional proper smooth variety defined over $\mathbb{Q}$.

In this paper we prove the first part of the Tate conjecture for $S_{K}$ for non-CM submotives if we assume that the field $F$ is Galois over $\mathbb{Q}$. We prove the second part of the Tate conjecture for $S_{K}$, without assuming that $F$ is Galois over $\mathbb{Q}$, but only for solvable number fields (see Theorem 8.2 for details). We remark that similar results were obtained by Ramakrishnan [12] in the case of Hilbert modular fourfolds and by Harder, Langlands, Rapoport [4], Murty, Ramakrishnan [11], Klingenberg [8], Lai [11] and Flicker and Hakim [3] in the case of Hilbert modular surfaces and compact quaternionic Shimura surfaces.

2. Quaternionic Shimura fourfolds and surfaces. Let $F$ be a totally real field of degree 4 over $\mathbb{Q}$ such that $F$ contains a quadratic number field $F_{0}$. We consider a quaternion division algebra $B$ over $\mathbb{Q}$ and let $D:=B \otimes_{\mathbb{Q}} F$. Assume that $D$ is a quaternion division algebra over $F$ which splits at the real places (we remark that given a quaternion division algebra $D$ over $F$ which splits at the real places, there exists a quaternion division algebra $B$ over $\mathbb{Q}$ such that $D:=B \otimes_{\mathbb{Q}} F$ if and only if for each rational prime $p$ we have $\sum_{v \mid p} \operatorname{inv}_{v} D_{v}=0$, where $v$ runs over the places of $F$ dividing $p$, and $\operatorname{inv}_{v}$ denotes the invariant of $D$ at $v$ ). Let $G$ be the algebraic group over $F$ defined by the multiplicative group $D^{\times}$. By restricting the scalars, we obtain the algebraic group $\bar{G}=\operatorname{Res}_{F / \mathbb{Q}}(G)$ over $\mathbb{Q}$ defined by the propriety $\bar{G}(A)=G\left(A \otimes_{\mathbb{Q}} F\right)$ for all $\mathbb{Q}$-algebras $A$.

Then, $\bar{G}(\mathbb{R})$ is isomorphic to $\mathrm{GL}_{2}(\mathbb{R})^{4}$. Let $h: \mathbb{C}^{*} \rightarrow \bar{G}(\mathbb{R})$ be defined by $a+b i \mapsto$ $\delta\left(\left(\begin{array}{cc}a & b \\ -b & a\end{array}\right)\right)$, where $\delta$ denotes the diagonal embedding of $\operatorname{GL}(2, \mathbb{R})$ in $\bar{G}(\mathbb{R})$. Let $K_{\infty}$ be the centralizer of $h$ in $\bar{G}(\mathbb{R})$. For each open compact subgroup $K \subset \bar{G}\left(\mathbb{A}_{f}\right)$ set

$$
S_{K}(\mathbb{C})=\bar{G}(\mathbb{Q}) \backslash \bar{G}\left(\mathbb{A}_{\mathbb{Q}}\right) / K K_{\infty}
$$

For $K$ sufficiently small, $S_{K}(\mathbb{C})$ is a complex manifold which is the set of the complex points of a proper smooth four-dimensional variety $S_{K}$ defined over $\mathbb{Q}$, which is called a compact quaternionic Shimura fourfold.

Let $D_{0}$ be a quaternion algebra over $F_{0}$ which splits at the real places such that $D=D_{0} \otimes_{F_{0}} F$ (we remark that $B \otimes_{\mathbb{Q}} F_{0}$ is a quaternionic division algebra over $F_{0}$ which has this propriety). Let $G_{0}$ be the algebraic group over $F_{0}$ defined by the multiplicative group $D_{0}^{\times}$. As above by restricting the scalars, we obtain the algebraic group 
$\bar{G}_{0}=\operatorname{Res}_{F_{0} / \mathbb{Q}}\left(G_{0}\right)$. Then, $\bar{G}_{0}(\mathbb{R})$ is isomorphic to $\mathrm{GL}_{2}(\mathbb{R})^{2}$. Let $h_{0}: \mathbb{C}^{*} \rightarrow \bar{G}_{0}(\mathbb{R})$ be defined by $a+b i \mapsto \delta_{0}\left(\left(\begin{array}{cc}a & b \\ -b & a\end{array}\right)\right)$, where $\delta_{0}$ denotes the diagonal embedding of $\operatorname{GL}(2, \mathbb{R})$ in $\bar{G}_{0}(\mathbb{R})$. Let $L_{\infty}$ be the centralizer of $h_{0}$ in $\bar{G}_{0}(\mathbb{R})$. For each open compact subgroup $L \subset \bar{G}_{0}\left(\mathbb{A}_{f}\right)$ set

$$
S_{0 L}(\mathbb{C})=\bar{G}_{0}(\mathbb{Q}) \backslash \bar{G}_{0}\left(\mathbb{A}_{\mathbb{Q}}\right) / L L_{\infty}
$$

For $L$ sufficiently small, $S_{0 L}(\mathbb{C})$ is a complex manifold which is the set of the complex points of a proper smooth two-dimensional variety $S_{0 L}$ defined over $\mathbb{Q}$, which is called a compact quaternionic Shimura surface.

3. Cohomologies for quaternionic Shimura fourfolds. Let $K$ be a sufficiently small open compact subgroup of $\bar{G}\left(\mathbb{A}_{f}\right)$.

If $l$ is a prime number, let $\mathbb{M}_{K}$ be the Hecke algebra generated by the bi- $K$-invariant $\overline{\mathbb{Q}}_{l}$-valued compactly supported functions on $\bar{G}\left(\mathbb{A}_{f}\right)$ under the convolution. If $\pi^{\prime}=$ $\pi_{f}^{\prime} \otimes \pi_{\infty}^{\prime}$ is an automorphic representation of $\bar{G}\left(\mathbb{A}_{\mathbb{Q}}\right)$, we denote by $\pi_{f}^{\prime K}$ the space of $K$-invariants in $\pi_{f}^{\prime}$. The Hecke algebra $\mathbb{H}_{K}$ acts on $\pi_{f}^{\prime K}$.

We have an action of the Hecke algebra $\mathbb{H}_{K}$ and an action of the Galois group $\Gamma_{\mathbb{Q}}$ on the étale cohomology $H_{\mathrm{et}}^{4}\left(S_{K}, \overline{\mathbb{Q}}_{l}\right)$ and these two actions commute. We say that the representation $\pi^{\prime}$ is cohomological if $H^{*}\left(\mathfrak{g}, \bar{G}_{\infty}, \pi_{\infty}^{\prime}\right) \neq 0$, where $\mathfrak{g}$ is the Lie algebra of $\bar{G}_{\infty}$ (the cohomology is taken with respect to the $\left(\mathfrak{g}, \bar{G}_{\infty}\right)$-module associated with $\left.\pi_{\infty}^{\prime}\right)$.

We know the following result (see for example Propositions 1.5 and 1.8 of [15]).

PROPOSITION 3.1. The representation of $\Gamma_{\mathbb{Q}} \times \mathbb{H}_{K}$ on the étale cohomology $H_{e t}^{4}\left(S_{K}, \overline{\mathbb{Q}}_{l}\right)(2)$ is isomorphic to

$$
\oplus_{\pi^{\prime}} \rho\left(\pi^{\prime}\right) \otimes \pi_{f}^{\prime K}
$$

where $\rho\left(\pi^{\prime}\right)$ is a representation of the Galois group $\Gamma_{\mathbb{Q}}$. The above sum is over weight 2 cohomological automorphic representations $\pi^{\prime}$ of $\bar{G}\left(\mathbb{A}_{\mathbb{Q}}\right)$, such that $\pi_{f}^{\prime K} \neq 0$, and the $\mathbb{H}_{K}$-representations $\pi_{f}^{\prime K}$ are irreducible and mutually inequivalent, i.e. the decomposition is isotypic with respect to the action of $\mathbb{}_{K}$.

The representations $\pi^{\prime}$ that appear in Proposition 3.1 are one dimensional or cuspidal and infinite dimensional. If $\pi^{\prime}$ is one dimensional, then $\rho\left(\pi^{\prime}\right)$ is six dimensional and if $\pi^{\prime}$ is cuspidal and infinite dimensional, then $\rho\left(\pi^{\prime}\right)$ is 16 dimensional. From now on in this paper we assume that $\pi^{\prime}$ is cuspidal and infinite dimensional, because for $\pi^{\prime}$ one dimensional the algebraicity of the Tate cycles corresponding to the $\pi^{\prime}$ component of $H_{e t}^{4}\left(S_{K}, \overline{\mathbb{Q}}_{l}\right)$ (see Proposition 3.1) could be proved in the same way as in Proposition 4.11 of [12], and the second part of the Tate conjecture in this case is also trivial (see [12]). We denote by $\pi$ the cuspidal automorphic representation of GL(2) $/ F$ corresponding to $\pi^{\prime}$ by Jaquet-Langlands correspondence.

Let $\rho_{\pi^{\prime}}=\rho_{\pi}$ be the $l$-adic two-dimensional semisimple representation of $\Gamma_{\mathbb{Q}}$ associated with $\pi^{\prime}$ or with $\pi$ (see $\left.[2, \mathbf{1 7}]\right)$. Then the representation $\rho\left(\pi^{\prime}\right)$ is semisimple (see section 7 of [13]) and $\rho\left(\pi^{\prime}\right)=\operatorname{As}_{F / \mathbb{Q}} \rho_{\pi^{\prime}}$, where $\operatorname{As}_{F / \mathbb{Q}} \rho_{\pi^{\prime}}$ is the Asai (or tensor induction) representation (see section 6 of [12]).

We fix an isomorphism $j: \overline{\mathbb{Q}}_{l} \rightarrow \mathbb{C}$ and define the $L$-function

$$
L^{4}\left(s, S_{K}\right):=\prod_{\pi^{\prime}} \prod_{q} \operatorname{det}\left(1-q^{-s+2} j\left(\rho\left(\pi^{\prime}\right)\left(\operatorname{Frob}_{q}\right)\right) \mid H_{\mathrm{et}}^{4}\left(S_{K}, \overline{\mathbb{Q}}_{l}\right)(2)^{I_{q}}\right)^{-1},
$$


where Frob $_{q}$ is a geometric Frobenius element at a rational prime $q$ and $I_{q}$ is a inertia group at $q$ (in order to define the local factor at $l$ one has to use actually the $l^{\prime}$-adic cohomology for some $l^{\prime} \neq l$ and Theorem 3 of [1] which gives us the expression of the local factors of the zeta functions of quaternionic Shimura varieties).

We have the canonical isomorphisms:

$$
\Phi_{K}: H_{B}^{4}\left(S_{K}(\mathbb{C}), \mathbb{Q}\right) \otimes_{\mathbb{Q}} \overline{\mathbb{Q}}_{l} \rightarrow H_{\mathrm{et}}^{4}\left(S_{K}, \overline{\mathbb{Q}}_{l}\right)
$$

and

$$
\Phi: H_{B}^{4}(S(\mathbb{C}), \mathbb{Q}) \otimes_{\mathbb{Q}} \overline{\mathbb{Q}}_{l} \rightarrow H_{\mathrm{et}}^{4}\left(S, \overline{\mathbb{Q}}_{l}\right),
$$

where $H_{B}^{4}\left(S_{K}(\mathbb{C}), \mathbb{Q}\right)$ is the Betti cohomology, and $S:=\lim _{\leftarrow} S_{K}$. We denote by $V\left(\pi^{\prime}\right)$ the $\pi^{\prime}$-component of $H_{\mathrm{et}}^{4}\left(S, \overline{\mathbb{Q}}_{l}\right)(2)$ in the decomposition of Proposition 3.1 and by $V_{B}\left(\pi^{\prime}\right)$ the corresponding $\pi^{\prime}$-component of $H_{B}^{4}(S(\mathbb{C}), \mathbb{Q})(2)$. Thus,

$$
V_{B}\left(\pi^{\prime}\right) \otimes_{\mathbb{Q}} \overline{\mathbb{Q}}_{l} \cong V\left(\pi^{\prime}\right) .
$$

Since $\rho\left(\pi^{\prime}\right)=\operatorname{As}_{F / \mathbb{Q}} \rho_{\pi^{\prime}}$, for each finite-order Hecke character $v$ of $F$, we have $\rho\left(\pi^{\prime}\right) \otimes$ $\left.v\right|_{I_{\mathbb{Q}}} \cong \rho\left(\pi^{\prime} \otimes v\right)$, where $I_{\mathbb{Q}}$ is the idele group of $\mathbb{Q}$, i.e. $\left.V\left(\pi^{\prime}\right) \otimes v\right|_{I_{\mathbb{Q}}} \cong V\left(\pi^{\prime} \otimes v\right)$ as $\Gamma_{\mathbb{Q}}$-modules.

4. Meromorphic continuation. For $\pi^{\prime}$ being a cuspidal representation as in Proposition 3.1, we denote by $A s_{F / F_{0}}\left(\pi^{\prime}\right)$ the isobaric automorphic representation of GL $\left(4, \mathbb{A}_{F_{0}}\right)$ defined in Theorem D of [14]. Let

$$
\rho_{A s_{F / F_{0}}\left(\pi^{\prime}\right)}: \Gamma_{F_{0}} \rightarrow \mathrm{GL}_{4}\left(\overline{\mathbb{Q}}_{l}\right)
$$

be the $l$-adic representation associated with $A s_{F / F_{0}}\left(\pi^{\prime}\right)$. Then, $\rho_{A s_{F / F_{0}}\left(\pi^{\prime}\right)}=A s_{F / F_{0}} \rho_{\pi^{\prime}}$ and $L\left(s, \rho_{A s_{F / F_{0}}\left(\pi^{\prime}\right)}\right)=L\left(s, A s_{F / F_{0}}\left(\pi^{\prime}\right)\right)$. From the proprieties of the Asai representations we know that $\rho\left(\pi^{\prime}\right)=A s_{F / \mathbb{Q}} \rho_{\pi^{\prime}}=A s_{F_{0} / \mathbb{Q}}\left(A s_{F / F_{0}} \rho_{\pi^{\prime}}\right)$, and because $A s_{F / F_{0}} \rho_{\pi^{\prime}}$ is automorphic, from Theorem 6.11 of [12], and using the solvable base change for GL(2) (see [10]) and the main theorem of [7], one obtains easily that (see also [1])

PROPOSITION 4.1. If $k / \mathbb{Q}$ is solvable, then the function $L\left(s,\left.\rho\left(\pi^{\prime}\right)\right|_{\Gamma_{k}}\right)$ has a meromorphic continuation to the entire complex plane and satisfies a functional equation $s \leftrightarrow 1-s$.

5. Some definitions. For $k$ being a number field, define

$$
\mathbf{V}\left(\pi^{\prime}, k\right):=\left\{x \in V\left(\pi^{\prime}\right) \mid \rho\left(\pi^{\prime}\right)(a) x=x \text { for all } a \in \Gamma_{k}\right\},
$$

and

$$
\mathbf{V}\left(\pi^{\prime}, \overline{\mathbb{Q}}\right):=\cup_{k} \mathbf{V}\left(\pi^{\prime}, k\right),
$$

where $V\left(\pi^{\prime}\right)$ is the space corresponding to $\rho\left(\pi^{\prime}\right)$. The elements of $\mathbf{V}\left(\pi^{\prime}, k\right)$ are called Tate cycles defined over $k$, and the elements of $\mathbf{V}\left(\pi^{\prime}, \overline{\mathbb{Q}}\right)$ are called Tate cycles. We denote by $\mathbf{U}\left(\pi^{\prime}, k\right) \subseteq \mathbf{V}\left(\pi^{\prime}, k\right)$ the subspace of algebraic cycles defined over $k$. 
We denote by $r_{\mathrm{alg}}\left(\pi^{\prime}, k\right):=\operatorname{dim}_{\overline{\mathbb{Q}}_{l}} \mathbf{U}\left(\pi^{\prime}, k\right)$, by $r_{l}\left(\pi^{\prime}, k\right):=\operatorname{dim}_{\overline{\mathbb{Q}}_{l}} \mathbf{V}\left(\pi^{\prime}, k\right)$, by $r_{l}\left(\pi^{\prime}, \overline{\mathbb{Q}}\right):=\operatorname{dim}_{\overline{\mathbb{Q}}} \mathbf{V}\left(\pi^{\prime}, \overline{\mathbb{Q}}\right)$ and for $k$ solvable number filed by $r_{a n}\left(\pi^{\prime}, k\right)$ the order of the pole of $L\left(s,\left.\rho\left(\pi^{\prime}\right)\right|_{\Gamma_{k}}\right)$ at $s=1$. Then, $r_{\mathrm{alg}}\left(\pi^{\prime}, k\right) \leq r_{l}\left(\pi^{\prime}, k\right)$.

For $v$ being a finite-order character of $\Gamma_{\mathbb{Q}}$, define

$$
\mathbf{V}\left(\pi^{\prime} ; v\right):=\left\{x \in V\left(\pi^{\prime}\right) \mid \rho\left(\pi^{\prime}\right)(a) x=v^{-1}(a) x \text { for all } a \in \Gamma_{\mathbb{Q}}\right\}
$$

and

$$
\mathbf{V}\left(\pi^{\prime}, \mathbb{Q}^{a b}\right):=\cup_{v} \mathbf{V}\left(\pi^{\prime} ; v\right) .
$$

Let $\mathbf{U}\left(\pi^{\prime} ; v\right) \subseteq \mathbf{V}\left(\pi^{\prime} ; v\right)$ and $\mathbf{U}\left(\pi^{\prime}, \mathbb{Q}^{a b}\right) \subseteq \mathbf{V}\left(\pi^{\prime}, \mathbb{Q}^{a b}\right)$ be the subspaces of algebraic cycles. We remark that when $\pi^{\prime}$ is non-CM, for $k$ sufficiently large we have $\mathbf{V}\left(\pi^{\prime}, k\right)=$ $\mathbf{V}\left(\pi^{\prime}, \mathbb{Q}^{a b}\right)$, i.e. all the Tate cycles are defined over abelian extensions of $\mathbb{Q}$. For $\pi^{\prime}$ of CM type, it is possible to have for all $k$ that $\mathbf{V}\left(\pi^{\prime}, k\right) \neq \mathbf{V}\left(\pi^{\prime}, \mathbb{Q}^{a b}\right)$.

We denote by $r_{\text {alg }}\left(\pi^{\prime} ; v\right):=\operatorname{dim}_{\overline{\mathbb{Q}}_{l}} \mathbf{U}\left(\pi^{\prime}, v\right)$, by $r_{l}\left(\pi^{\prime} ; v\right):=\operatorname{dim}_{\overline{\mathbb{Q}}_{l}} \mathbf{V}\left(\pi^{\prime}, v\right)$ and by $r_{a n}\left(\pi^{\prime}, v\right)$ the order of the pole of $L\left(s, \rho\left(\pi^{\prime}\right) \otimes v\right)$ at $s=1$. Then, $r_{\text {alg }}\left(\pi^{\prime} ; \nu\right) \leq r_{l}\left(\pi^{\prime} ; \nu\right)$, $r_{l}\left(\pi^{\prime}, \mathbb{Q}^{a b}\right) \leq r_{l}\left(\pi^{\prime}, \overline{\mathbb{Q}}\right)$.

6. Matching Tate cycles and poles. We say that an automorphic representation $\pi$ of GL(2)/ $F$ for some number field $F$ is of CM type if there exists some quadratic Galois character $\eta: I_{F} / F^{\times} \rightarrow \overline{\mathbb{Q}}_{l}^{\times}$, with $\eta \neq 1$ such that $\pi \cong \pi \otimes \eta$. We say that a representation $\rho$ of a group $G$ is dihedral if there exists a normal subgroup $N$ of index 2 in $G$ and a character $\chi: N \rightarrow \mathbb{C}^{\times}$such that $\rho=\operatorname{Ind}_{N}^{G} \chi$. If $\pi$ is an automorphic representation of GL(2)/F as in Proposition 3.1, then $\pi$ is of CM type if and only if $\rho_{\pi}$ is a dihedral representation. If $\pi$ corresponds to an automorphic representation $\pi^{\prime}$ of $\bar{G}\left(\mathbb{A}_{f}\right)$ by Jacquet-Langlands correspondence, then we say that $\pi^{\prime}$ is CM if $\pi$ is CM.

Using in particular the decomposition (in some cases) of $\rho\left(\pi^{\prime}\right)$ as a sum of automorphic representations, more exactly as a direct sum of $l$-adic representations associated Hecke characters and of twists by Hecke characters of $\mathrm{Sym}^{2} \pi^{\prime \prime}$ and $\mathrm{Sym}^{4} \pi^{\prime \prime}$ for non-CM representations $\pi^{\prime \prime}$ of GL(2) (which from [5] and [8] we know that are cuspidal and irreducible), in [12] (Propositions 8.6 and 8.8) are proved the following two lemmas (for the definition of $\pi^{\prime}$ and $\pi$ see Proposition 3.1 and the comments after it):

LEMMA 6.1. For $\pi^{\prime}$ non-CM, all the Tate classes in $V\left(\pi^{\prime}\right)$ are rational over an abelian number field $k$, with

$$
r_{l}\left(\pi^{\prime}, k\right) \leq 2
$$

hence,

$$
r_{l}\left(\pi^{\prime}, \mathbb{Q}^{a b}\right)=r_{l}\left(\pi^{\prime}, \overline{\mathbb{Q}}\right) \leq 2
$$

Lemma 6.2. Let $F / \mathbb{Q}$ be Galois, and $\pi^{\prime}$ non-CM. Then

(a) $r_{l}\left(\pi^{\prime}, \mathbb{Q}^{a b}\right) \neq 0$ iff a twist of $\pi$ is a base change from a quadratic subextension of $F$.

(b) $r_{l}\left(\pi^{\prime}, \mathbb{Q}^{a b}\right)=2$ iff a twist of $\pi$ is a base change from $\mathbb{Q}$. 
(c) The following are equivalent:

(i) $r_{l}\left(\pi^{\prime} ; v\right)=2$ for some $v$.

(ii) A twist of $\pi$ is a base change from $\mathbb{Q}$, and $F$ is biquadratic.

From Lemma 6.1 above and section 8 of [12] we know that

Proposition 6.3. For $\pi^{\prime}$ non- $C M$ we have

$$
r_{l}\left(\pi^{\prime} ; \nu\right)=r_{a n}\left(\pi^{\prime} ; \nu\right) \leq 2,
$$

and thus because $r_{\text {alg }}\left(\pi^{\prime} ; v\right) \leq r_{l}\left(\pi^{\prime} ; v\right)$, we have

$$
r_{a l g}\left(\pi^{\prime} ; v\right) \leq r_{l}\left(\pi^{\prime} ; v\right)=r_{a n}\left(\pi^{\prime} ; v\right) \leq 2
$$

We also know that (see Proposition 8.5 of [12])

Proposition 6.4. If $\pi^{\prime}$ is of CM type, we have for any $k$,

$$
r_{l}\left(\pi^{\prime}, k\right)=r_{a n}\left(\pi^{\prime}, k\right) .
$$

7. Twisted Hirzebruch-Zagier cycles. We use the same notations as in section 2, i.e. we consider a quaternion division algebra $D_{0}$ over some quadratic subextension $F_{0}$ of $F$ such that $D=D_{0} \otimes_{F_{0}} F$. Then, the map $h$ factors through the map $h_{0}$ of $R_{\mathbb{C} / \mathbb{B}}\left(\mathbb{C}^{*}\right)$ into $\bar{G}_{0 \mathbb{R}}$. The natural diagonal embedding of $\bar{G}_{0}$ into $\bar{G}$ defines a morphism

$$
\delta_{L, K}: S_{0 L} \hookrightarrow S_{K}
$$

over $\mathbb{Q}$, if $L$ is contained in $K$.

For any $g \in \bar{G}\left(\mathbb{A}_{f}\right)$, and any open compact subgroup $K$ of $\bar{G}\left(\mathbb{A}_{f}\right)$, define the corresponding Hirzebruch-Zagier cycle (or $H-Z$ cycle) (relative to $\bar{G}_{0}$ ) to be the algebraic cycle of codimension 2 of $S_{K}$ given by

$$
{ }^{D_{0}} Z_{g, K}=R(g)\left(\delta_{\bar{G}_{0}\left(A_{f}\right) \cap g K g^{-1}, g K g^{-1}}\left(S_{0 \bar{G}_{0}\left(\mathrm{~A}_{f}\right) \cap g K g^{-1}}\right)\right),
$$

where $R(g): S_{g K g^{-1}} \rightarrow S_{K}$ is the right translation action on Shimura varieties.

Now for each character of finite order $\mu$ of $F$, we have the usual twisted correspondence $R(\mu) \subset S_{K} \times S_{K[\mu]}$, where $K[\mu]$ is some level which depends on $K$ and $\mu$ (see for example section 5 of [12] for details). This twisting correspondence is algebraic and acts on any cohomology group, Betti or étale, of the fourfold $S=\lim S_{K}$. The induced operator sends the $\pi^{\prime}$-component to the $\pi^{\prime} \otimes \mu$-component. The twisting correspondence $R(\mu)$ is rational over $\mathbb{Q}\left(\mu_{1}\right)$, where $\mu_{1}=\left.\mu\right|_{I_{\mathbb{Q}}}$, and $I_{\mathbb{Q}}$ is the idele group of $\mathbb{Q}$.

For each character of finite order $\mu$ of $F$ and each H-Z cycle $Z$ on $S$, let $Z(\mu)$ be the $\mu$-twisted $H-Z$ cycle obtained by pushing forward $Z$ under $R(\mu)$. Then, $Z(\mu)$ is algebraic and rational over $\mathbb{Q}\left(\mu_{1}\right)$.

8. Matching algebraic cycles and poles. We prove

PROPOSITION 8.1. Let $F$ be a quartic, Galois, totally real number field, and $\pi^{\prime}$ be a non-CM cuspidal automorphic representation of $\bar{G}\left(\mathbb{A}_{\mathbb{Q}}\right)$ of weight 2 that appears in 
Proposition 3.1. Then for any character of finite order $v$ of $\Gamma_{\mathbb{Q}}$, we have

$$
r_{a l g}\left(\pi^{\prime} ; \nu\right)=r_{a n}\left(\pi^{\prime} ; \nu\right)
$$

Proof. From Proposition 6.3 we know that $r_{\text {alg }}\left(\pi^{\prime} ; \nu\right) \leq r_{a n}\left(\pi^{\prime} ; \nu\right) \leq 2$.

We distinguish three cases:

(A) $r_{a n}\left(\pi^{\prime} ; v\right)=0$. Then $r_{\text {alg }}\left(\pi^{\prime} ; v\right)=0$ and we are done.

(B) $r_{a n}\left(\pi^{\prime} ; v\right)=1$. Then as in the proof of Theorem 9.1 of [12], one can find a quadratic subfield $F_{1}$ of $F$ and a finite-order character $\mu$ of $F$ such that $r_{\text {alg }}\left(\pi^{\prime} ; \nu\right)=$ $r_{\mathrm{alg}}\left(\pi^{\prime} \otimes \mu ; 1\right)$ and such that $L\left(s, A s_{F / F_{1}}\left(\pi^{\prime} \otimes \mu\right)\right)=L\left(s, A s_{F / F_{1}}(\pi \otimes \mu)\right)$ has a simple pole at $s=1$, which by the residue formula of [4] implies that there exists some function $\phi$ in the space of $\pi$ such that

$$
\int_{\mathrm{GL}\left(2, F_{1}\right) Z\left(\mathbb{A}_{F_{1}}\right) \backslash \mathrm{GL}\left(2, \mathbb{A}_{F_{1}}\right)} \phi(g) \mu(\operatorname{det}(g)) d g \neq 0,
$$

where $Z$ denotes the centre of GL(2). From [6] (the main theorem) and [3] (the appendix), we deduce that that there exists some function $\phi^{\prime}$ in the space of $\pi^{\prime}$ such that

$$
\int_{\bar{G}_{1}(\mathbb{Q}) \bar{Z}_{1}\left(\mathbb{A}_{\mathbb{Q}}\right) \backslash \bar{G}_{1}\left(\mathrm{~A}_{\mathbb{Q}}\right)} \phi^{\prime}(g) \mu(\operatorname{det}(g)) d g \neq 0,
$$

where $\bar{Z}_{1}$ denotes the centre of $\bar{G}_{1}=\operatorname{Res}_{F_{1} / \mathbb{Q}}\left(G_{1}\right)$, and $G_{1}$ is the algebraic group over $F_{1}$ defined by the multiplicative group $D_{1}^{\times}$of a suitable quaternion division algebra $D_{1}$ over $F_{1}$ which satisfies that $D=D_{1} \otimes_{F_{1}} F$ (more exactly let $\mathcal{S}$ be the set of places $v$ of $F_{1}$ which split into two different places $w$ and $\bar{w}$ of $F$ such that $D_{w}$ and $D_{\bar{w}}$ are ramified (we remark that because $D=B \otimes_{\mathbb{Q}} F$, we get that $B \otimes_{\mathbb{Q}} F_{1}$ is a quaternion division algebra over $F_{1}$, and thus we have that for each two different places $w$ and $\bar{w}$ of $F$ dividing a place $v$ of $F_{1}, D_{w}$ and $D_{\bar{w}}$ have the same invariant). If $|\mathcal{S}|$ is even, then there exists a quaternion division algebra $D_{1}$ over $F_{1}$ which ramifies at exactly the places $v$ in $\mathcal{S}$ such that $D=D_{1} \otimes_{F_{1}} F$. Then by the main theorem of [6], $D_{1}$ satisfies the above propriety. If $|\mathcal{S}|$ is odd, then from [3] (appendix) we know that there exists a place (actually infinitely many) $v_{1}$ of $F_{1}$ outside $S$ which does not split into $F$ and a quaternion division algebra $D_{1}$ over $F_{1}$ which is ramified at exactly the places $v$ in $\mathcal{S} \cup v_{1}$, such that $D=D_{1} \otimes_{F_{1}} F$ and has the above propriety). Hence, the integral of a $(2,2)$-form $\eta_{\phi^{\prime}}$ on the compact quaternionic Shimura fourfold $S_{K}$ defined by $\phi^{\prime}$ has a non-zero $\mu$-twisted period over a Hecke translate of the embedded compact quaternionic Shimura surface attached to $D_{1}$. Thus, the corresponding twisting self-correspondence of the fourfold defines for some $g \in \bar{G}\left(\mathbb{A}_{f}\right)$ a $\mu$-twisted H-Z cycle $Z(\mu)={ }^{D_{1}} Z_{g, K}(\mu)$ of codimension 2 such that

$$
\int_{Z(\mu)} \eta_{\phi^{\prime}} \neq 0
$$

and hence $Z(\mu)$ is homologically non-trivial. Thus, $r_{\mathrm{alg}}\left(\pi^{\prime} ; \nu\right) \geq 1$, and we obtain that $r_{\mathrm{alg}}\left(\pi^{\prime} ; \nu\right)=1$, and we are done.

(C) $r_{a n}\left(\pi^{\prime} ; \nu\right)=2$. From part (c) of Lemma 6.2 we deduce that $F / \mathbb{Q}$ is biquadratic, and then as in [12], one can find a finite-order character $\mu$ of $F$ such that $r_{\text {alg }}\left(\pi^{\prime} ; \nu\right)=$ $r_{\text {alg }}\left(\pi^{\prime} \otimes \mu ; 1\right)$ and such that $\pi \otimes \mu$ is a base change from two quadratic subfields $F_{1}$ and 
$F_{2}$ of $F$. Then as in (B) because the functions $L\left(s, A s_{F / F_{1}}\left(\pi^{\prime} \otimes \mu\right)\right)$ and $L\left(s, A s_{F / F_{2}}\left(\pi^{\prime} \otimes\right.\right.$ $\mu)$ ) have simple poles at $s=1$, we get, for some quaternion algebras $D_{1}$ and $D_{2}$ over $F_{1}$ and $F_{2}$ and some $g_{1}, g_{2} \in \bar{G}\left(\mathbb{A}_{f}\right)$, two twisted codimension 2 algebraic cycles $Z_{1}:={ }^{D_{1}}$ $Z_{g_{1}, K}(\mu)$ and $Z_{2}:={ }^{D_{2}} Z_{g_{2}, K}(\mu)$ on $S$ which are homologically non-trivial because the period integrals of some $(2,2)$-forms over these cycles are non-zero. But these two cycles could be proportional in the $\pi^{\prime}$-component of the cohomology, and thus one may have to replace one of them by a twisted version. Then in [12], Lemma 9.3, a finite-order character $\xi$ of $F$ is defined such that it has some special signature at the infinite places, and such that $\left.\xi\right|_{I_{F_{1}}}=1$, and thus $\left.\xi\right|_{I_{\mathbb{Q}}}=1$ and hence

$$
r_{a n}\left(\pi^{\prime} \otimes \xi ; \nu\right)=r_{a n}\left(\pi^{\prime} ; v\right) .
$$

Also $L\left(s, A s_{F / F_{1}}\left(\pi^{\prime} \otimes \xi \mu\right)\right)$ has a simple pole at $s=1$, and thus, if we define

$$
Z_{3}:={ }^{D_{1}} Z_{g_{3}, K}^{*}(\mu \xi)
$$

we have for some $g_{3} \in \bar{G}\left(\mathbb{A}_{f}\right)$, and some $\phi^{\prime}$ in the space of $\pi^{\prime}$ that

$$
\int_{Z_{3}} \eta_{\phi^{\prime}} \neq 0
$$

Then, from Lemma 9.4 of [12], we know by looking at the signatures at infinite places of the classes of $Z_{1}, Z_{2}$ and $Z_{3}$ in $V_{B}\left(\pi^{\prime}\right)$ that the space spanned by the classes of $Z_{1}, Z_{2}$ and $Z_{3}$ in $V_{B}\left(\pi^{\prime}\right)$ has dimension 2 . Thus, $r_{\mathrm{alg}}\left(\pi^{\prime} ; v\right) \geq 2$ and we obtain that $r_{\text {alg }}\left(\pi^{\prime} ; \nu\right)=2$, and we are done.

We can deduce now the following result.

THEOREM 8.2. Let $F$ be a quartic totally real number field containing a quadratic subfield. Let $\pi^{\prime}$ be an automorphic representation as in Proposition 3.1. Then,

(a) For any solvable number field $k$, the function $L\left(s,\left.\rho\left(\pi^{\prime}\right)\right|_{\Gamma_{k}}\right)$ has a meromorphic continuation to the complex plane and satisfies a functional equation $s \leftrightarrow 1-s$.

(b) If $F / \mathbb{Q}$ is Galois, then for any solvable number field $k$ we have that $\operatorname{dim}_{\overline{\mathbb{Q}}_{l}} \mathbf{V}\left(\pi^{\prime}, k\right)$ is equal to the order of the pole of the function $L\left(s,\left.\rho\left(\pi^{\prime}\right)\right|_{\Gamma_{k}}\right)$ at $s=1$. If $\pi^{\prime}$ is $C M$, this result is true for any number field $k$.

(c) If $F / \mathbb{Q}$ is Galois and $\pi^{\prime}$ is non-CM, then for any number field $k$ we have

$$
\operatorname{dim}_{\overline{\mathbb{Q}}_{l}} \mathbf{U}\left(\pi^{\prime}, k\right)=\operatorname{dim}_{\overline{\mathbb{Q}}_{l}} \mathbf{V}\left(\pi^{\prime}, k\right) .
$$

Proof. Part (a) is the statement of Proposition 4.1. Now assume that $F / \mathbb{Q}$ is Galois and $\pi^{\prime}$ is non-CM. Then from Propositions 6.3 and 8.1 we get that $r_{\text {alg }}\left(\pi^{\prime} ; \nu\right)=$ $r_{l}\left(\pi^{\prime} ; v\right)=r_{a n}\left(\pi^{\prime} ; v\right) \leq 2$, and from Lemma 6.1 we deduce part (c). Now if $\pi^{\prime}$ is CM, part (b) is the statement of Proposition 6.4. For $\pi^{\prime}$ non-CM and $k$ solvable we know from (a) that $L\left(s,\left.\rho\left(\pi^{\prime}\right)\right|_{\Gamma_{k}}\right)$ has a meromorphic continuation to the complex plane, and we have to match the order of the pole of $L\left(s,\left.\rho\left(\pi^{\prime}\right)\right|_{\Gamma_{k}}\right)$ at $s=1$ with the dimension of the space of the Tate cycles defined over $k$. But because $k$ is solvable and $\pi^{\prime}$ is non-CM we get that $\left.\rho_{\pi^{\prime}}\right|_{\Gamma_{F k}}$ is cuspidal irreducible. Now because $K$ contains a quadratic subextension $F_{0}$ we get that $\left.\rho\left(\pi^{\prime}\right)\right|_{\Gamma_{k}}$ is a tensor product of Asai representations of degree 4, 2 or 1 associated with cuspidal representations of GL(2). When we have degree 4, i.e $\left.\rho\left(\pi^{\prime}\right)\right|_{\Gamma_{k}}=A s_{F k / k} \rho_{\pi^{\prime \prime}}$, for some cuspidal non-CM automorphic representation $\pi^{\prime \prime}$ of GL(2)/Fk (associated with $\left.\rho_{\pi^{\prime}}\right|_{\Gamma_{F k}}$ ) and $F k$ has a quadratic subextension $k^{\prime} / k$, 
we obtain part (b) exactly as in [12], section 8 (it is proved in the same way as Proposition 6.3). The rest of the cases are similar.

\section{REFERENCES}

1. D. Blasius, Hilbert modular forms and the Ramanujan conjecture, in Noncommutative geometry and number theory (Aspects Math. vol. E37) (Vieweg, Wiesbaden, 2006) 35-56.

2. H. Carayol, Sur la mauvaise réduction des courbes de Shimura, Compositio Mathematica 59(2) (1986), 151-230.

3. Y. Z. Flicker and J. L. Hakim, Quaternionic distinguished representations, Am. J. Math. 116(3) (June 1994), 683-736.

4. G. Harder, R. P. Langlands and M. Rapoport, Algebraische Zycklen auf HilbertBlumenthal-Flächen, J. Reine Angew. Math. 366 (1986), 53-120.

5. H. Jacquet and S. Gelbart, A relation between automorphic representations of $G L(2)$ and GL(3), Ann. Sci. École Norm. Sup. 11 (1979), 471-542.

6. H. Jacquet and K. Lai, A relative trace formula, Compositio Math. 54 (1985), 243-310.

7. H. Jacquet, I. I. Piatetski-Shapiro and J. A. Shalika, Rankin-Selberg convolutions, Am. J. Math. 105(2) (1983), 367-464.

8. C. Klingenberg, Die Tate-Vermutungen für Hilbert-Blumenthal-Flächen, Invent. Math. 89 (1987), 291-317.

9. K. F. Lai, Algebraic cycles on compact Shimura surface, Math. Z. 189 (1985), 593-602.

10. R. P. Langlands, Base change for GL(2) (Ann. Math. Studies vol. 96) (Princeton University Press, Princeton, NJ, 1980).

11. V. K. Murty and D. Ramakrishnan, Period relations and the Tate conjecture for Hilbert modular surfaces, Invent. Math. 89 (1987), 319-345.

12. D. Ramakrishnan, Algebraic cycles on Hilbert modular fourfolds and poles of $L$ functions, in Proceedings of the International Conference on Algebraic Groups and Arithmetic (2005), 271-274.

13. D. Ramakrishnan, Modular curves, modular surfaces and modular fourfolds, in Algebraic cycles and motives, Volume 1, 278-292, dedicated to Jacob Murre, Cambridge University Press, London Math. Soc. Lecture Notes 343 (2007).

14. D. Ramakrishnan, Modularity of solvable Artin representations of GO(4)-type, Int. Math. Res. Not. (1) (2002), 1-54.

15. J. D. Rogawski and J. B. Tunnell, On Artin $L$-functions associated to Hilbert modular forms of weight one, Invent. Math. 74 (1983), 1-43.

16. J. Tate, Algebraic cycles and poles of zeta functions, in Arithmetical algebraic geometry (Schilling O. D. G., Editor) (Harper and Row, New York, 1966).

17. R. Taylor, On Galois representations associated to Hilbert modular forms, Invent. Math. 98 (1989), 265-280. 